This article was published as: Sensors and actuators A: Physical 248, 54-61, 2016 DOI: http://dx.doi.org/10.1016/j.sna.2016.06.020 


\title{
MECHANOELECTRICAL TRANSDUCTION IN THE HYDROGEL-BASED BIOMIMETIC SENSORS
}

\section{F.A. Blyakhman ${ }^{\text {a,b,*, A.P. Safronov }}{ }^{\text {c }}$, A.Yu. Zubarev ${ }^{\text {d, T.F. Shklyar }}{ }^{\text {a,b }}$, O.A. Dinislamova ${ }^{\text {a }}$,} M.T. Lopez-Lopez ${ }^{\text {e }}$

a Department of biomedical physics and engineering, Ural State Medical University, 3 Repin Str., Yekaterinburg 620028, Russian Federation

b Department of Physics, Ural Federal University named after the first President of Russia B.N.Yeltsyn, Yekaterinburg 620083, Russian Federation

${ }^{c}$ Department of Chemistry, Ural Federal University named after the first President of Russia B.N.Yeltsyn, Yekaterinburg 620083, Russian Federation

${ }^{\mathrm{d}}$ Department of Mathematics, Ural Federal University named after the first President of Russia B.N.Yeltsyn, Yekaterinburg 620083, Russian Federation

${ }^{\text {e }}$ Departamento de F1sica Aplicada, Universidad de Granada, 18071 Granada, Spain

*corresponding author

E-mail addresses: Feliks.Blyakhman@urfu.ru (Felix Blyakhman), Biomedical Physics Department, Ural State Medical University, 3 Repin Str., Yekaterinburg 620028, Russian Federation; phone: +7 (912) 2885776

\begin{abstract}
The study addresses the phenomenon of mechanoelectrical transduction in the polyelectrolyte hydrogels and, in particular, the search of driving force for the change of gel electrical potential under applied mechanical stretch. Polyelectrolyte gels of calcium and magnesium salts of polymethacrylic acid were synthesized by the radical polymerization in water solution. Their electrical potential measured by microcapillary electrodes was negative and fall within $100-140$ $\mathrm{mV}$ range depending on the nature of counterion and gel networking density. The rectangular samples $(\sim 10 \mathrm{~mm}$ in length and $2 \times 2 \mathrm{~mm}$ in cross-section) of gel-based sensors underwent the dynamic axial deformation, and simultaneous monitoring of their geometrical dimensions and the electrical potential was performed. Sensor elongation resulted in the overall increase of gel volume, and it always was accompanied by the change of gel potential toward the depolarization (diminishing of the negative values). Theoretical model based on the assumption of total electrical charge constancy in the course of filament dynamic deformation was proposed to describe the dependence of gel potential on its volume. Good agreement between the model predictions and the experimental trend was shown. The proposed mechanism of mechanoelectrical transduction based on the stretch-dependant volume change of polyelectrolyte hydrogels might be useful to understand the nature of mechanical sensing in much more complex biological gels like the cell cytoskeleton.
\end{abstract}

Keywords: biomimetic sensors, polyelectrolyte hydrogel, electrical potential, mechanical deformation, depolarization, modeling 


\section{Introduction}

The synthetic polymeric hydrogels widely introduced as biomimetic material for the biomedical applications. From the general viewpoint of physical chemistry, the cell, especially the cell cytoskeleton, structurally resembles a polyelectrolyte hydrogel. The gel is a 3D cross-linked polymer network with the electric charges localized on the macromolecular filaments, and with free counterions dispersed in the liquid phase inside the network [Harland and Prudhomme, 1992]. The physical basis of mechanical contraction and expansion of polyelectrolyte hydrogels stem from the balance of interaction of polymeric filaments with the medium, the entropic flexibility of filaments, the positive osmotic pressure of counterions, and the balance of forces of attraction and repulsion between electric charges (Hirotsu, 1993).

Basically, the chemical stricture and key interaction mechanisms of proteins networking in a living cell are much complex from synthetic hydrogels. Meanwhile, the close similarity between these two physical systems in their structural properties and in the response to some basic external stimuli was demonstrated in numerous studies [1-3] including some performed by us [4-6].

In particular, the cells and anionic gels are negatively charged reference to their surroundings, and they both keep electrical potential close to $-100 \mathrm{mV}$. The negative electrical potential of anionic polyelectrolyte gel is a direct result of Donnan equilibrium established on the gel/supernatant boundary [3,4,7]. According to IUPAC nomenclature [8], Donnan equilibrium takes place if one or more ionic species for some particular reason can not cross the phase boundary while other ionic species can freely move across it.

One of the most striking and important phenomena in the cell is the mechanoelectrical transduction (MET). In general, it combines the influence of the mechanical deformation on the electrical potential in living cells $[9,10]$. The most clear manifestation of MET is the functioning of the excitable cells, especially the muscle cells. In particular, in the cardiomyocytes, where cyclic deformation takes place, MET plays extremely important role in the determining of heart rate pattern [11].

Meanwhile, recently we have demonstrated the existence of MET in the synthetic polyelectrolyte gels of calcium and magnesium salts of polyacrylic acid [12]. We have shown that the deformation of a gel filament in an axial direction results in the decrease of the gel negative potential. We suppose that one of the most reasonable physical mechanisms, which can explain this phenomenon, is the possibility of gel volume changes during deformation. As the gel is immersed in water, the condition of its constant volume is not applicable as water molecules can enter and exit the interior of gel. Consequently, the gel network extra swelling or contraction may result in the 
changes in counterions concentration in polymer matrix, which may affect the gel electrical potential. Indirectly, the results of theoretical [13,14] and experimental [15] studies that demonstrated the effects of polyelectrolyte hydrogel free swelling on its electrical potential may support to proposed hypothesis.

The objective of the present study was to reveal the correlation between the stretching deformation, the volume changes, and the electrical potential of gel-based sensors. The experimental setup included application of the dynamic axial deformations to the sensors with simultaneous monitoring of their linear dimensions, volume and the electrical potential. Based on these experiments we intended to develop a theoretical model for the MET phenomenon in the synthetic hydrogel filament in the ionic solution, which hopefully might be applicable to the wide range of gel systems.

\section{Experimental part}

\subsection{Materials}

Polyelectrolyte gels based on poly(methacrylic) acid (PMA) with calcium and magnesium counterions (CaPMA and MgPMA) were synthesized by free-radical polymerization in $2.7 \mathrm{M}$ aqueous solution. Prior to polymerization monomeric methacrylic acid (Merck, Schuchardt, Honenbrunn) was completely neutralized by the addition of stoichiometric amount of magnesium or calcium oxides. N,N'-methylene-diacrylamide was used as a cross-linker. Cross-linker concentration was set at 1:50, 1:100, 1:200, 1:400 to monomer concentration in molar ratio, which further on is named as the network density of the gel. Subsequent gels are further denoted as $\mathrm{Ca}(\mathrm{Mg}) \mathrm{PMAn}$, where $\mathrm{n}$ stands for the number of monomer units per one cross-link. Polymerization was carried out in polyethylene probe tubes, $10 \mathrm{~mm}$ in diameter, at $800 \mathrm{C}$ for 2 hours using ammonium persulfate as initiator.

After the synthesis, the gels were washed in daily renewed distilled water for two weeks to remove non-reacted monomers, salts, and linear olygomers. The actual content of ionized groups in gels was determined by means of the thermogravimetry (Netzsch STA409 thermal analyzer) by measuring the residual weight after heating of previously dried gels up to $8000 \mathrm{C}$ at a heat rate 10 $\mathrm{K} / \mathrm{min}$ in the air. During the heating, the gels were decomposed to water, carbon dioxide and the residue of metal oxide. The stoichiometric equation of decomposition gave metal content in the synthesized gel. The content of ionized carboxylate groups was 98\% (mol) in CaPMA gels and 75\% in MgPMA gels. The synthesis of polymers and their swelling behavior are described in our early 
publications in details [4,5]. To avoid some structural inhomogeneity of proposed sensors, the central part of gel cylinders was cut by a razor blade to elongated rectangular samples, $10 \mathrm{~mm}$ in length and approximately $2 \times 2 \mathrm{~mm}$ in cross-section.

\subsection{Experimental equipment}

Fig. 1 schematically displays the experimental setup of laboratory design used in this study. The equipment was built around an optical system, and contained a thermostatic bath for the gel sample, a semiconductor force transducer, an electromagnetic motor providing mechanical deformations, a semiconductor optical transducer measuring the motor's lever displacement, a personal computer equipped for controlling the experiment.

The electrical potential of a gel sensor was obtained by two identical $\mathrm{Ag} / \mathrm{AgCl}$ tapered glass microelectrodes $(\sim 1$ micron in tip diameter) typically used in biophysical studies for intracellular voltage measurement. The details of Donnan potential measurement in the gel were described in several studies $[3,4,7]$. Briefly, thin-walled, single-barrel borosilicate capillary tubes "TW150F-6" ("World Precision Instruments", USA) were single-pulled using a standard electrode puller "ME-3" ("EMIB Ltd", Russia). The pulled electrodes were immersed in a $3 \mathrm{M} \mathrm{KCl}$ solution with the tip facing upward, so that the solution climbed to the tip by capillary action.

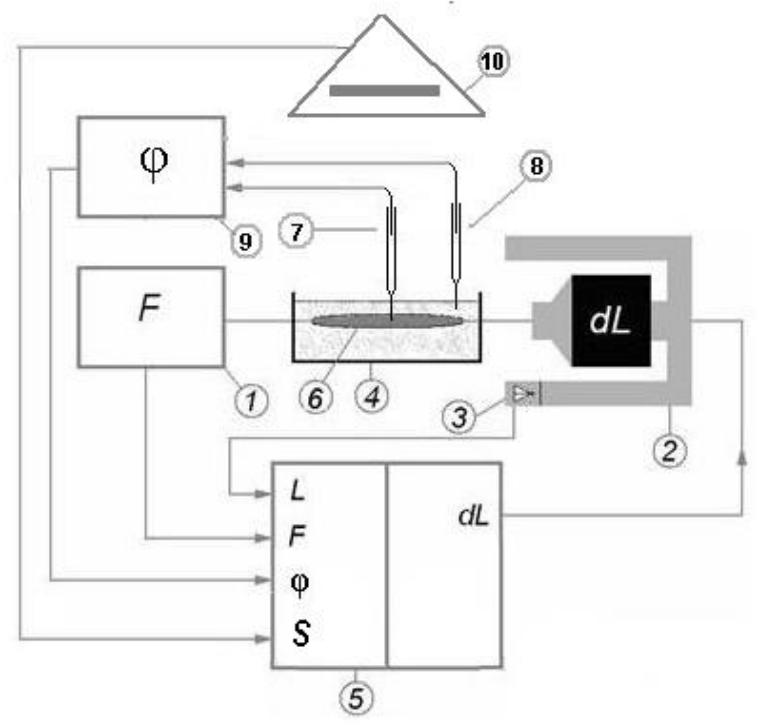

Fig. 1. Scheme of the experimental setup. 1 - force transducer, 2 - electromagnetic motor, 3 length transducer, 4 - experimental bath, 5 - computer, 6 - gel sample, 7,8 - glass-micropipette electrodes, 9 - instrumental amplifier, 10 - digital video camera. 
One electrode was pinned into the gel sample, the other was placed in water outside. The potential difference between microelectrodes was measured using an instrumental amplifier on the base of an integrated circuit "INA 129" ("Burr-Brown", USA). The main amplifier parameters were: input impedance $-10^{10} \mathrm{Ohm}$, frequency bandwidth $-0 \ldots 107 \mathrm{~Hz}$, gain -50 . To reduce the influence of electromagnetic interference on the potential difference measurement, special wire shields were provided around the unit. Typically, the peak-to-peak noise at the output of instrumental amplifier during the gel potential monitoring was no higher of $5 \mathrm{mV}$.

Computerized optical system on the base of digital video camera ("Panasonic" HX-WA2) was used for the monitoring of gel sensor planar projection. The camera was placed beneath of the bath's transparent bottom, and video files were recorded.

\subsection{Experimental protocol}

All experiments were performed at room temperature on the gels immersed in the distilled water. One end of a rectangular gel sensor $\sim 10 \mathrm{~mm}$ in length and $\sim 2 \mathrm{~mm}$ in width was affixed to the force transducer or stationary lever, while the other end was connected to the linear motor. With use the generator of signals based on specially written software, the stepwise and oscillatory triangular linear axial deformations were applied to the gel by means of the linear motor (see Fig. 1). At first, the electrochemical (Donnan) potential $(\varphi)$ of the gel was measured at every sample length stationary level after the deformation step. When triangular deformations with the rate from $0.4^{*} 10^{-}$ ${ }^{4}$ to $1.6^{*} 10^{-4}$ meter per second were used, the dynamic changes of gel's length, planar projection and potential were recorded simultaneously. The value of the deformation $(\varepsilon)$ was defined as the relative length change of the gel sample: $\varepsilon=L i / L o$. To calculate the volume of gel sensor, the contours of samples were outlined by hand using the specially written software. The evolution of gel's length and width was performed with $\sim 1.0 \mathrm{sec}$ interval.

\subsection{Statistical analysis}

The statistic software package StatSoft was used for the statistical analysis. Mean and standard deviation (SD) of the measured parameters were calculated. To estimate the interrelation between measured values the correlation tests and the regression analysis were performed.

\section{Results}


Fig. 2 shows the typical experimental plot of the electrical potential $(\varphi)$ measurement in a polyelectrolyte MgPMA50 gel by a capillary microelectrode. The baseline with $\varphi=0$ corresponds to allocation of electrode in a supernatant solution outside the gel sample. Upon pinning the capillary electrode into the gel, its potential dropped down to the value ca. $-120 \mathrm{mV}$, which remained constant. When the electrode was taken out, $\varphi$ returned to the baseline.

The negative electrical potential of anionic polyelectrolyte gel is a result of the difference in concentration of counterions inside and outside the gel. The concentration of $\left[\mathrm{Mg}^{2+}\right]$ counterions inside the negatively charged gel network is higher than in the surrounding water medium; therefore, the measured electrochemical potential of the gel is negative.

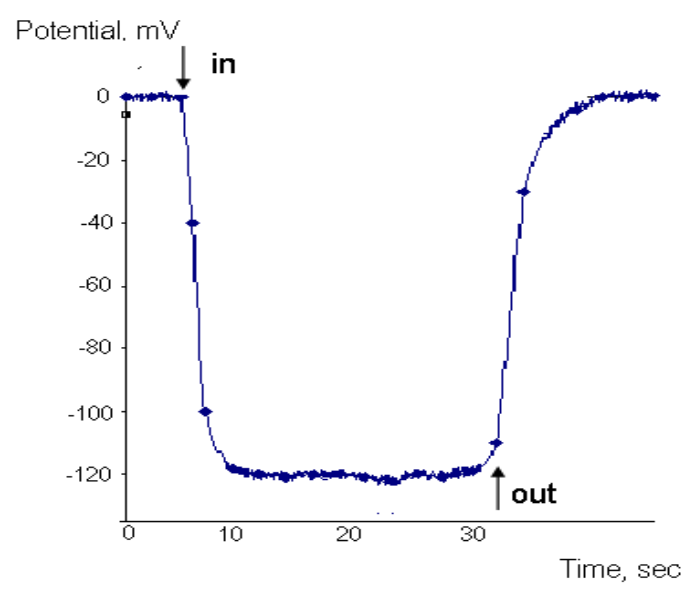

Fig. 2. Typical experimental plot of potential measurement on the MgPMA50 gel sensor.

Fig. 3 shows the dependence of gel potential on the nature of divalent counterions $\left(\mathrm{Ca}^{++}\right.$, $\mathrm{Mg}^{++}$) and on the gel network density. The potential negative values of MgPMA gels are twice higher than that of CaPMA gels. The extent of gel network charge density is the reason for obtained result. As is known, the dissociation degree of calcium salts is in general lower than that of magnesium salts [Саша, на кого сослаться?]. Hence, the negative electrical potential of CaPMA gel is also lower.

In addition, Fig. 3 demonstrates a distinct diminishing trend in the potential of both gel series with the increase in network density. Although the difference between mean values of potential for CaPMA gels is not significant, the MgPMA50 hydrogels are statistically different respectively others. Meanwhile, the close correlation between the potential and network density for both series of gels was found: correlation coefficients 0.98 and 0.97 for CaPMA and MgPMA 
samples, respectively. Obtained result implies that the lower of the gel density the higher of the gel potential negative value, and it completely fits with data presented early [,].Саша, может что-то напишешь почему так?

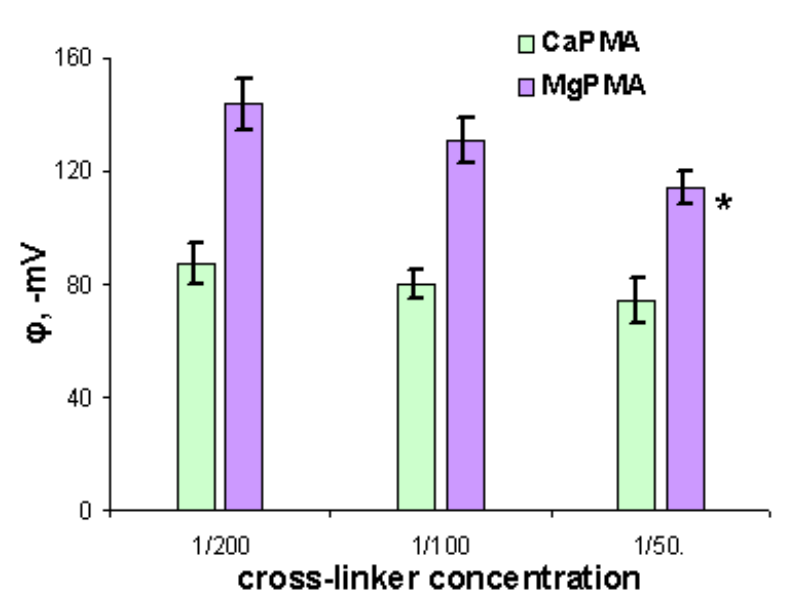

Fig. 3. Potential of CaPMA and MgPMA hydrogels in non-deformed state. Cross-linker concentration presented as the number of monomer units per one cross-link. Error bars mark standard deviation of the potential mean value $(n=6)$. Asterisk demonstrates the significant difference between MgPMA50 and MgPMA100 with P<0.05; MgPMA50 and MgPMA200 samples with $\mathrm{P}<0.01$

The periodical mechanical deformation applied to the gel sensor changed its potential. Fig. 4 shows the time course of triangular linear axial deformation applied to the gel sample and the corresponding gel potential measured simultaneously. During the stretch of a sample, initial negative value of the gel potential shifted toward the depolarization. It means that the gel became less negatively charged. During the release phase of the deformation, the restoration of gel potential took place. The depolarization - restoration cycles closely followed the stretch - release cycles. 


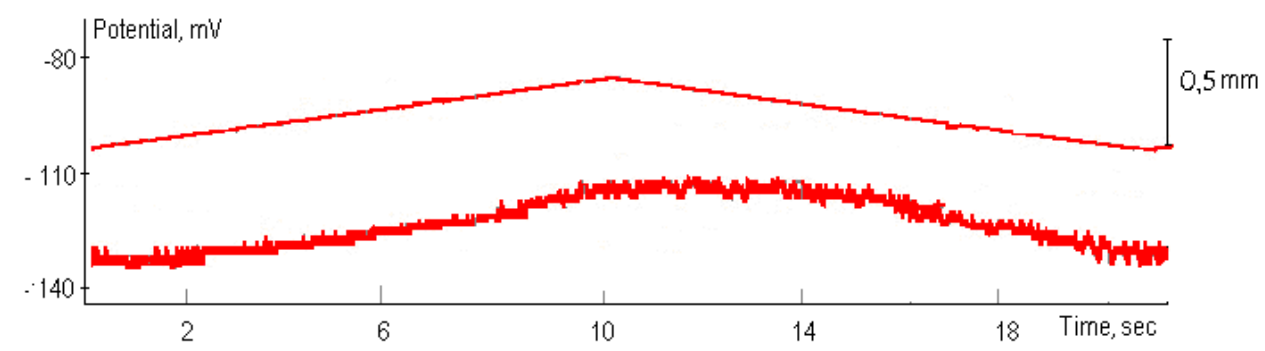

Fig. 4. Typical experimental record of the MgPMA100 gel potential under applied cyclic deformation. Upper curve corresponds to the output of optical transducer, which measured the motor lever displacement. Lower curve is the output of instrumental amplifier, which measured the gel sensor potential simultaneously. The scale bar on the right corresponds to the sample length change.

Table 1 presents the result of gel potential measurements obtained in MgPMA200 sensors in the course of step-wise deformation. Mean values $(n=7)$ and SD of potential at different extent of sample stretch are presented. One can see that the elongation of the gel sensors resulted in the decrease of negative values of the potential. Close inverse correlation between the gel potential and the gel stretch was found $(r=-0.99)$.

\section{Table 1}

Dependence of the gel sensor (MgPMA200) potential on the applied stretch.

\begin{tabular}{|l|l|}
\hline Deformation, Li/Lo & Potential, $\mathrm{mV}$ \\
\hline 1,00 & $-123 \pm 4$ \\
\hline 1,05 & $-119 \pm 5$ \\
\hline 1,10 & $-113 \pm 3$ \\
\hline 1,15 & $-104 \pm 6$ \\
\hline 1,20 & $-100 \pm 3$ \\
\hline
\end{tabular}


The length and the width of the gel filament were monitored during the deformation cycles, and Fig. 5 shows the time dependence of their values related to the non-deformed state. At the phase of the applied stretch in $5 \mathrm{sec}$, the length of sensor increased 1.7 times, while the relative width decreased only to 0.9 . At the phase of sample release, its length decreased and the width increased back. It is evident from Fig. 5 that the increase in relative length of the sensor is much larger than the decrease in its width. It means that the volume of filament does not keep constant during deformation cycles.

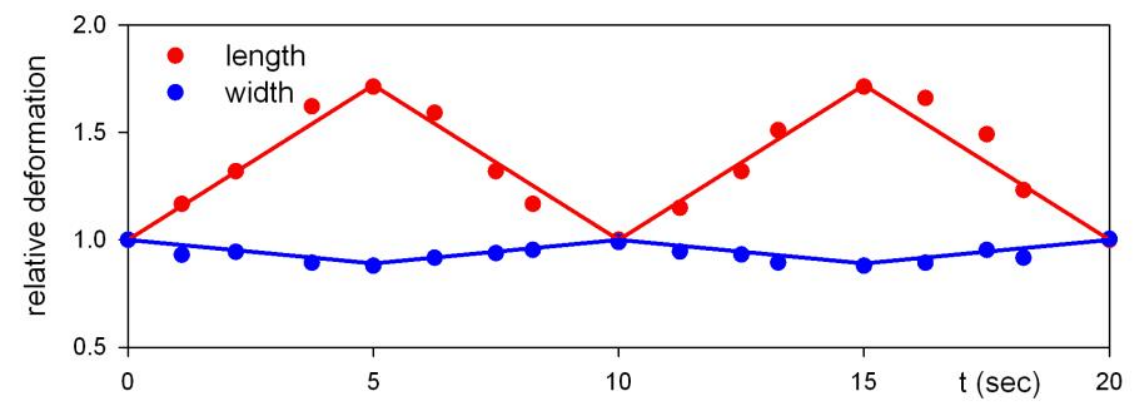

Fig. 5. Dynamics of the gel sensor (MgPMA200) length and width under the applied triangular linear axial deformations.

The estimation of filament volume as the product of its length and square of the width revealed that the volume increased at the stage of stretch application and decreased at the release stage. Fig. 6 presents the generalized dependence of the relative volume changes of the gel filament upon its relative elongation due to applied stretch. Mean values and SD of relative volume change at the maximum of applied stretch for several CaPMA and MgPMA gel-based sensors are plotted.

It is clear that the volume of the gel filament does not stay constant if elongating deformation is applied. The dependence appeared to be rather universal in the series of gels studied in the present work. Within the experimental error, the relative volume changes for MgPMA and CaPMA gels with different network density fit the same curve up to $25 \%$ stretch of samples. It shows a close to a linear raise, and tends to saturation at higher elongations of CaPMA gel sensors with network density of 1:200 and 1:400. The volume increase is rather substantial: it is more than $10 \%$ at $20 \%$ stretch. 


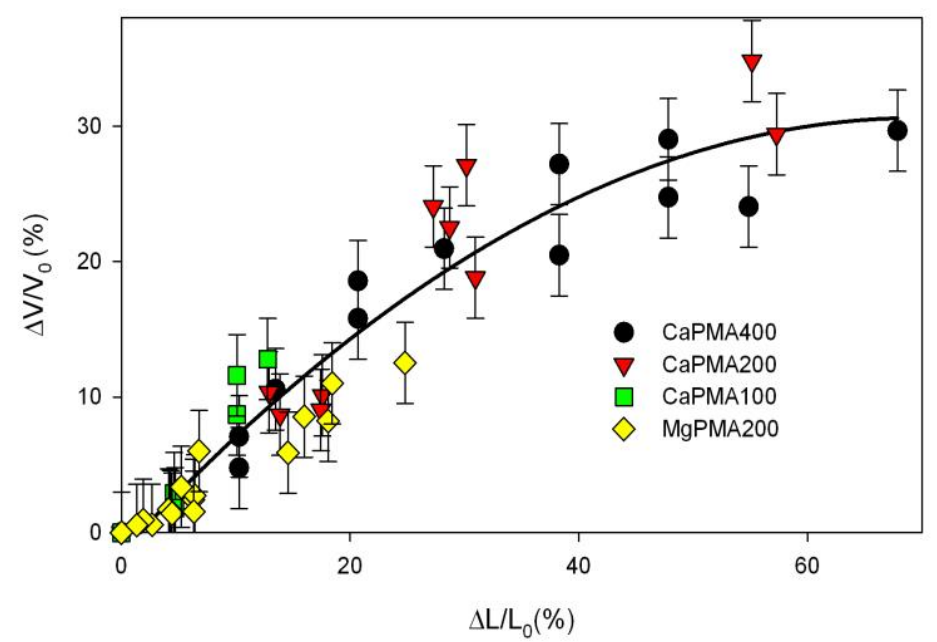

Fig. 6. Dependence of sensor volume changes under stretch.

It looks reasonable that such induced swelling of a gel upon its deformation can provide the consequent changes in its electrical potential. To disclose possible interrelation between these factors we introduce the following theoretical model.

\subsection{Model}

Андрей, может здесь надо что-то добавить, типа, не смотря на то, что в эксперименте использовались прямоугольные образцы... В методике я объясню, почему прямоугольные. Кроме того, вставь все по месту, что ты написал в ответах на критику, пожалуйста! For maximal simplification of mathematics, we will consider a cylindrical polymeric gel filament with the length $L$ which is much larger than its radius $R$. The cylindrical model of the sample allows us to get the final results in mathematically and physically transparent form and keeps all physically principal points of the experimental situation. The filament is placed in an infinitive liquid with the dielectric permeability $\varepsilon$.

In our experiments, the monovalent negative ions $\mathrm{COO}^{-}$are fixed on the polyelectrolyte chains which constitute the gel network; movable counterions $\mathrm{Mg}^{++}$are distributed inside and outside the filament. Let us denote the total negative electrical charge, the gel chains carry on, as $Q$.

The supernatant liquid outside the filament contains $\mathrm{Mg}^{++}$counterions, which form a double electrical layer around the filament. Besides, the aqueous supernatant contains $\mathrm{H}^{+}$and $\mathrm{OH}^{-}$ions 
originated from the self dissociation of water molecules. At the infinite distance from the filament, the electroneutrality condition is maintained.

It is evident from Fig. 5 that the width of filament slightly decreases if the filament is stretched. However, the decrease is small and for the sake of simplicity we took the radius of the model filament being constant during deformation. This condition is not crucial for the model, which can easily be modified to incorporate the slight decrease of the radius as well.

Our aim is to find the mean, over the filament cross-section, magnitude $\langle\varphi\rangle$ of the electrical potential inside the filament as a function of its length. The considered model is illustrated in Fig. 7.

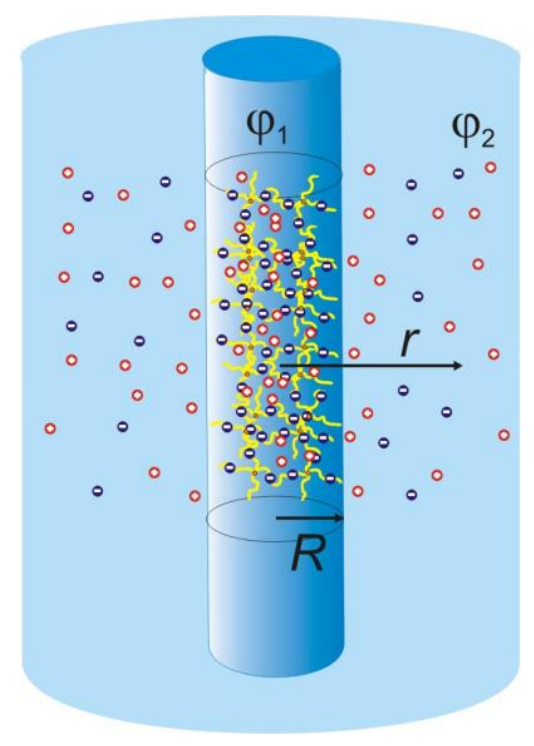

Fig. 7. Sketch of the model under consideration

Let us denote $\rho_{1}=\frac{\mathrm{Q}}{\pi \mathrm{R}^{2} L}>0$ (Андрей, что-то надо объяснить здесь, наверное) the absolute magnitude of the mean charge density inside the filament; the local charge density outside the fiber we will denote as $\rho_{2}(r)$, where $r$ is a distance from the cylinder axis (see Fig. 7).

The electrical potentials $\varphi 1$ and $\varphi 2$ inside and outside the filament respectively, satisfy the Poisson equations

$$
\nabla^{2} \varphi_{1}=\frac{4 \pi}{\varepsilon} \rho_{1}, \quad r<R
$$

and 


$$
\nabla^{2} \varphi_{2}=\frac{4 \pi}{\varepsilon} \rho_{2}, \quad r>R
$$

Inside the filament, the electrical charge consists of the negative charge directly situated on the polymer chains as well as on the charges of the motile positive and negative ions. We will suppose that the total charge of the ions fixed on the chains is not changed during the elongation of the filament.

In contrast, the charge, provided by the motile ions, can alternate due to the change of the condition of thermodynamic equilibrium between the ions inside and outside the filament. Here it is worth noting that the total charge of the polyelectrolyte filament with all the counterions should be zero to obey the electroneutrality condition. However, this condition is not strictly fulfilled in the layers adherent to the surface of the filament as counterions are free to move to the supernatant. Therefore, these layers carry on non-compensated negative charge. Usually, the law of the fixed ions spatial distribution inside the polymer filament is unknown. We suppose, for simplicity, that this distribution is homogeneous, i.e. it does not depend on $r$. This assumption corresponds to the fact that the filament is optically transparent. That means that this is structurally homogeneous. The motile ions inside and outside the filament obey the Boltzmann law of distribution.

Taking it into account in the Debye - Huckel approximation $q_{ \pm} \varphi_{1,2}<<k T$, by using (2), we get the following equation for the potential outside the filament:

$$
\nabla^{2} \varphi_{2}=\kappa^{2} \varphi_{2}
$$

Here $\kappa=\sqrt{\frac{4 \pi}{\varepsilon k T}\left(q_{+}^{2} n_{+}+q_{-}^{2} n_{-}\right)}$is the inverse Debye thickness of the double electrical layer, $\mathrm{n} \pm$ and $q_{ \pm}$ are the concentrations of the positive and negative ions infinitely far from the filament and their charges respectively. One can show easily that in the framework of the Debye - Huckel approximation the charge density of the motile ions is significantly less than that of the charges fixed on the chains and can be neglected. In the framework of this approximation the charge density inside the filament coincides with the uniform density of the fixed charges $\rho 1$.

Taking it into account we get the following solutions of the equations (1) and (3):

$$
\begin{aligned}
& \varphi_{1}=\frac{\pi}{\varepsilon} \rho_{1} r^{2}+A \\
& \varphi_{2}=B H_{0}(\kappa r)
\end{aligned}
$$


Here $H_{0}(\mathrm{x})$ is the modified Hankel function, $A$ and $B$ are constants to be determined.

Their magnitudes can be found from the following standard boundary relations of electrostatics:

$$
r=R, \varphi_{1}=\varphi_{1}, \frac{\partial \varphi_{1}}{\partial r}=\frac{\partial \varphi_{2}}{\partial r}
$$

Here we use the simplification that the dielectric permeability $\varepsilon$ inside and outside the polymer filament is the same.

Combining eqs. (4) and (5) we get:

$$
\begin{aligned}
& \varphi_{1}(r)=\frac{\pi}{\varepsilon} \rho_{1}\left[r^{2}-R^{2}+2 \frac{R H}{\kappa H^{\prime}}\right] \\
& H=H_{0}(\kappa R), \quad H^{\prime}=\left.\frac{d H_{0}(x)}{d x}\right|_{x=\kappa R}
\end{aligned}
$$

The average, over the fiber cross-section, electrical potential is:

$$
<\varphi_{1}>=\frac{2}{R^{2}} \int_{0}^{R} \varphi_{1}(r) d r=-\frac{\pi}{\varepsilon}\left[\frac{1}{2} R^{2}-2 \frac{R H}{\kappa H^{\prime}}\right]
$$

Let us note that the Hankel function, being positive, monotonously decreases, therefore the inequality $H^{\prime}<0$ is held. Thus, the relation in the square brackets of (7) is positive.

For the majority of the typical situations the strong inequality $\kappa R>>1$ is fulfilled. By using the well-known asymptotic expressions for the Hankel function, in this limiting case we have:

$$
H=\frac{\exp (-\kappa R)}{\sqrt{\kappa R}}, \quad H^{\prime}=-\frac{1}{2} \frac{\exp (-\kappa R)}{(\kappa R)^{3 / 2}}(1+2 \kappa R) \approx-H
$$

By using this estimate in (7), one can get

$$
<\varphi_{1}>=-\frac{\pi}{\varepsilon} \rho_{1}\left[\frac{1}{2} R^{2}-2 \frac{R}{\kappa}\right] \approx-\frac{\pi}{2 \varepsilon} \rho_{1} R^{2}
$$

Let $\rho_{10}$ be that charge density inside the fiber when its length is $L_{0}$. Since the total charge $Q$ of the filament is permanent, the following relation

$$
\rho_{1}(L)=\rho_{10} \frac{L_{0}}{L}
$$


is held. By using (9) in eqs. (7) or (8), one can see, that absolute magnitude of the negative average potential $\left\langle\varphi_{1}\right\rangle$ decreases while the fiber length $L$ increases. This conclusion, at least qualitatively, corresponds to the trend of diminishing of the magnitude of gel potential observed in experiment.

The quantitative agreement is surprisingly also good. As we do not have actual data on the magnitude of $Q$, we have estimated the value $\frac{\pi}{2 \varepsilon} \rho_{10} R^{2}$ from the condition that the estimate (8) fits the experimental results for $L=L_{0}$.

Fig. 8 shows the calculated dependence of the potential on the stretch of gel filament given by solid line in comparison with the experimental data presented in Table 1. It is seen that the model curve nicely fits experimental points.

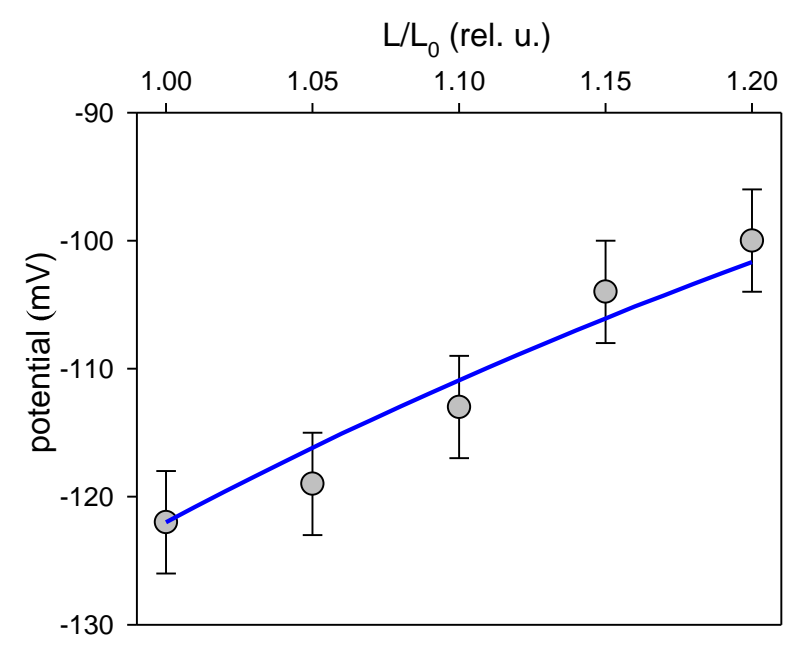

Fig. 8. Dependence of the potential of MgPMA200 gel on the applied stretch. Line corresponds to the fitting of experimental points by the model Equation (8).

\section{Discussion}

This work is the result of our long-term studies addressed the electrical and mechanical properties of polyelectrolyte hydrogels based on the poly(acrylic) and poly(methacrylic) acids. The effects of many factors, such as the degree of gel swelling, the composition and ionic strength of surrounding solution, the type of counterions, the gel network density on the gel potential and gel viscoelasticity were defined and reported in a series of our publications [,]. Besides, we 
demonstrated the existence of MET in the gels [,]. We showed that at a given deformation velocity, the extent of gel deformation closely correlates with the gel potential. Furthermore, we found that at the same level of gel deformation, the lower the deformation velocity, the higher is the gel potential relative change.

Finally, the gel samples of CaPMA and MgPMA with the network density of 1:200-400 were chosen as most suitable prototypes of the gel mechanoelectrical sensor with the sensitivity of 1 $-2 \mathrm{mV}$ per $1 \%$ of deformation depended on the network density and the regime of stretching [,]. According to mechanical examination of the gels used [,], the stress of $3-5 \mathrm{~N} / \mathrm{m}^{2}$ resulted in $1 \%$ of gel sample stretching deformation.(Ребята, прикиньте корректность фразы про напряжение, МЮ у этих гелей 3-5 КПА)

The main point of present investigation is a search for reasonable mechanism to explain the MET in hydrogels-based sensors. Possible impact of gel stretch-dependant volume change on the gel potential was our working hypothesis. To test this, the advanced experiments with simultaneous measurements of the gel volume and gel potential in the course of applied deformation, and theoretical model were performed and developed. Given the good correlation between the model predictions and the actual mechanoelectrical transduction in polyelectrolyte filament, we may turn to the possible physical reasons which underlie the electrical response of the synthetic hydrogel to the mechanical stimuli.

The ability of polymeric network to swell in a liquid under the applied stretching deformation was one of the first theoretical results of the classic Flory theory of polymer solutions experimentally confirmed for some swollen rubber materials [15,16]. In general, a polymeric network tends to swell in the plane across the direction of stretching. The basic reason is the tendency of a polymeric subchain to keep its equilibrium conformation of a random Gaussian coil. The applied force stretches the coils of subchains along the direction of its action. The subproduct of this stretching is the compression of polymeric coils in a plane across this direction under the normal stresses. As the compression is thermodynamically unfavorable, the random coil tends to reestablish equilibrium dimensions by the absorption of a solvent and swelling. In case of polyelectrolyte network this general trend is enforced by electrostatic contribution, which is illustrated in Fig. 9. For the sake of simplicity, the process is shown in a step-wise fashion. 


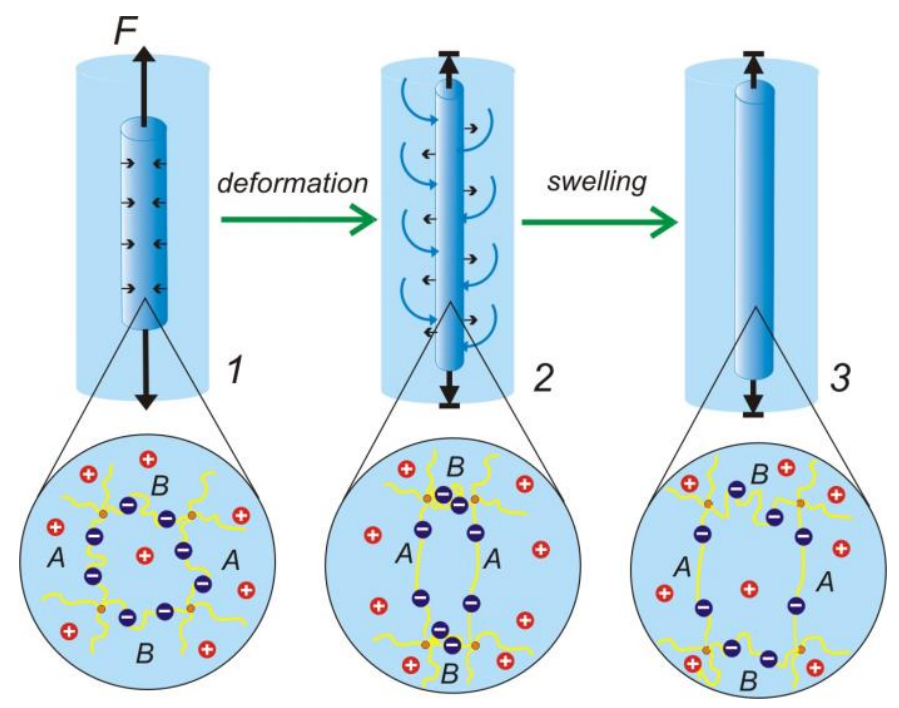

Fig. 9. Scheme of gel swelling induced by its deformation. Upper row - macroscopic forces and fluxes. Lower row - microscopic view on polymer network. 1 - initial structure of gel, 2 - gel structure under strain; volume remains constant, 3 - gel structure under strain; volume increased due to the swelling. $A$ - polymeric subchains oriented along the direction of elongation. $B-$ polymeric subchains oriented across the direction of elongation.

Mark 1 in Fig. 9 corresponds to the initial network structure of polyelectrolyte gel, which is to be deformed by the applied stress. Let us define two types of polymeric subchains between the cross-links, which constitute the network. Subchains marked $A$ are positioned along the applied force, subchains marked B are oriented across the direction of elongation. The non-deformed network structure is isotropic: both subsets of polymeric subchains are statistically equivalent. Their conformation is close to statistical coil. There are negatively charged residues affixed at the subchains and the equivalent number of positively charged counterions, which compensate the electrical charge of the chains. If elongating stress is applied, the normal stresses appear at the walls of the sample, and lead to the contraction of the gel across the direction of elongation.

Mark 2 in Fig. 9 shows the result of the elongating deformation if the volume of the gel remained constant. The network structure becomes anisotropic: while subchains $A$, which carry the load, stretch along the direction of deformation, subchains $B$ compress due to the applied normal stresses. The compressed compact conformation of subchains $B$ is unfavorable both as it deviates from the conformation of a random coil and as the distance between adjacent residues diminishes and the electrostatic repulsion between them increases. To recover the conformation of a statistical coil and to decrease the electrostatic repulsion in subchains $B$ gel absorbs water from the supernatant and swells. Mark 3 in Fig. 9 shows that due to the swelling of gel across the direction of 
elongation subchains $B$ uncurl and the distance between negatively charged residues allocated on them increases.

The increase of the gel volume due to its swelling results in the diminishing of the total charge density of the polymeric network. Effectively, the network becomes less charged. It seems reasonable to conclude that the negative potential of the gel will also diminish; i.e. the gel will depolarize. The model developed above rigorously confirms this intuitively clear inference.

Meanwhile, there are certain limitations in both the experimental setup and the theoretical modeling. For instance, we have supposed that such processes as the dissociation of polar groups in the network, the condensation of counterions, and the ionic equilibrium at the surface are not affected by the stretching deformation. It might be true at a short time scale but the condition of static ionic distribution in the stretched gel will certainly be violated in long time intervals. Good correlation between the values of the potential measured and calculated in the model show that at the characteristic time scale of the dynamic stretching, which was around $5 \mathrm{sec}$ for each consequent phase, ionic distribution in the gel filament apparently stayed constant.

Based on these considerations one would then expect the reverse polarization of the permanently stretched gel in a long time interval due to the slow relaxation to the equilibrium ionic distribution.

\section{Conclusions}

By the consistent measurements of the geometrical dimensions and the electrical potential of the gel sensor under the dynamic periodical stretching deformation we have found that the filament swells at the elongating step and deswells at the release step. Simultaneously the negative values of gel potential diminish if gel filament swells due to its stretching and increase at gel contraction. The dependence of the potential upon gel volume is adequately described by a theoretical model based on the assumption of the constancy of the total electrical charge of the gel filament in the course of dynamic deformation. The quantitative agreement between experimental and calculated values of the potential is achieved.

Although the experiment was restricted to the synthetic polyelectrolyte gels of calcium and magnesium salts of polymethacrylic acid, we believe that the same general mechanism of mechanoelectrical transduction might be useful to understand the nature of mechanical sensing in much more complex biological gels like cell cytoskeleton. As is well known, the key role of the cytoskeleton sub-membrane structures (cortex) to provide for MET is attributed to all eukaryotic 
cells (Chalfie, 2009; Baumgarten, 2007; Hamill and Martinac, 2001; Sachs, 2010). Based on the concept that general physical laws should apply both in living and non-living matter, we may assume an important role of the cytoskeleton physicochemical properties in the controlling of cell potential. Independently of biological mechanisms complexity, the polyelectrolyte hydrogels might certainly be biophysically advantageous for the construction of biocompatible gel-based devices, which may be widely introduced as the biomimetic sensors of strain, stress, and stretch.

\section{Acknowledgment}

This work has been done under the financial support of the Russian Scientific Fund, project 14-19-00989. One of us (M.T. Lopez-Lopez) has been supported by the Grant FIS2013-41821-R (MINECO, Spain).

\section{References}

[1] G.N. Ling, Revolution in the Physiology of the Living Cell, Krieger Pub, Co., Malabar, 1992.

[2] G.H. Pollack, Cells, Gels and the Engines of Life, Ebner\&Sons, Seattle, 2001.

[3] R.W. Guelch, J. Holdenried, A. Weible, T. Wallmersperger, B. Kroeplin, Electroactive Polymer Actuators and Devices, Proceedings of the SPIE 3987 (2000) 193-202.

[4] A.P. Safronov, T.F. Shklyar, V. Borodin, Y. A. Smirnova, S.Yu. Sokolov, G.H. Pollack, F.A. Blyakhman, Donnan Potential in Hydrogels of Poly(Methacrylic Acid) and Its Potassium Salt, Water in Biology, G. Pollack, I. Cameron, D. Wheatley, Springer, 2006, pp. 273-284.

[5] T. F. Shklyar, A.P. Safronov, I.S. Klyuzhin, G.H. Pollack, F.A. Blyakhman. A Correlation between Mechanical and Electrical Properties of the Synthetic Hydrogel chosen as an Experimental Model of Cytoskeleton, Biophysics 53 (2008) 544-549.

[6] T.F. Shklyar, A.P. Safronov, O.A. Toropova, G.H. Pollack, F.A. Blyakhman, Mechanical Characteristics of Synthetic Polyelectrolyte Gel as a Physical Model of the Cytoskeleton, Biophysics 56 (2011) 68-73.

[7] F. Gao, F.B. Reitz, G.H. Pollack, Potentials in Anionic Polyelectrolyte Hydrogels. J. Appl. Polym. Sci. 89 (2003) 1319-1321.

[8] IUPAC Compendium of Chemical Terminology, 1997. 
[9] O.P. Hamill, B. Martinac, Molecular Basis of Mechanotransduction in Living Cells, Physiological Reviews 81 (2001) 685-740.

[10] P.G. Gillespie, R.G. Walker. Molecular basis of mechanosensory transduction, Nature 413 (2001) 194-202;

[11] T.A. Quinn, P. Kohl, U. Ravens, Cardiac Mechano-electric Coupling Research: Fifty Years of Progress and Scientific Innovation, Prog. Biophys. Mol. Biol. 115 (2014) 71-75.

[12] T.F. Shklyar, O.A. Dinislamova, A.P. Safronov, F.A. Blyakhman, Effect of Cytoskeletal Elastic Properties on the Mechanoelectrical Transduction in Excitable Cells, J. Biomech. 45 (2012) 1444-1449.

[13] L. Feng, Y. Jia, X. Chen, X. Li, L. An, A Multiphasic Model for the Volume Change of Polyelectrolyte Hydrogels, J. Chem. Phys., 133 (2010) 114904.

[14] L. Feng, Y. Jia, X. Li, L. An, Comparison of the Multiphasic Model and the Transport Model for the Swelling and Deformation of Polyelectrolyte Hydrogels, J. Mech. Behav. Biomed. 4 (2011) 1328-1335.

[15] P.J. Flory, J. Rehner, Statistical Mechanics of Cross-Linked Polymer Networks II Swelling, J. Chem. Phys. 11 (1943) 521-526.

[16] L.R.G. Treloar, The swelling of cross-linked amorphous polymers under strain, Transactions of the Faraday Society 46 (1950) 783-789.

1. Harland, R. S., Prudhomme., R. K., 1992. Polyelectrolyte Gels: Properties, Preparation and Applications. In: Harland, R. S., Prudhomme., R. K. (Eds). Polyelectrolyte Gels: Properties, Preparation and Applications. American Chemical Society, Washington, DC, pp. 450.

2. Hirotsu S., 1993. Responsive gels: volume transitions II, Advances in polymer sciences. In: Dusek, K. (Eds), Springer-Verlag, Berlin, pp. 275. 


\section{Figure captions}

Fig. 1. Scheme of the experimental setup. 1 - force transducer, 2 - electromagnetic motor, 3 length transducer, 4 - experimental bath, 5 - computer, 6 - gel sample, 7,8 - glass-micropipette electrodes, 9 - instrumental amplifier, 10 - digital video camera.

Fig. 2. Typical experimental plot of potential measurement on the MgPMA50 gel sensor.

Fig. 3. Potential of CaPMA and MgPMA hydrogels in non-deformed state.

Fig. 4. Typical experimental record of the MgPMA100 gel potential under applied cyclic deformation. Upper curve corresponds to the output of optical transducer, which measured the motor lever displacement. Lower curve is the output of instrumental amplifier, which measured the gel sensor potential simultaneously.

Fig. 5. Dynamics of the gel sensor length and width under the applied triangular linear axial deformations.

Fig. 6. Dependence of sensor volume changes under stretch.

Fig. 7. Sketch of the model under consideration

Fig. 8. Dependence of the potential of MgPMA200 gel on the applied stretch. Line corresponds to the fitting of experimental points by the model Equation (8).

Fig. 9. Scheme of gel swelling induced by its deformation. Upper row - macroscopic forces and fluxes. Lower row - microscopic view on polymer network. 1 - initial structure of gel, 2 - gel structure under strain; volume remains constant, 3 - gel structure under strain; volume increased due to the swelling. $A-$ polymeric subchains oriented along the direction of elongation. $B-$ polymeric subchains oriented across the direction of elongation. 